\title{
Los patrones electroforéticos de proteínas salivales permiten diferenciar los grupos transandino y cisandino de las especies de Rhodnius de Colombia
}

Arlid Meneses, Cristian Camilo Rodríguez, Yazmín Suárez, Julio César Carranza, Gustavo Adolfo Vallejo

Laboratorio de Investigaciones en Parasitología Tropical, Universidad del Tolima, Ibagué, Colombia

Introducción. Las especies Rhodnius (Hemiptera: Reduviidae: Triatominae) están conformadas por insectos hematófagos vectores de Trypanosoma cruzi, agente etiológico de la enfermedad de Chagas, y T. rangeli, parásito infectivo pero no patógeno para el vertebrado. El estudio de la diversidad proteica de la saliva de estos insectos permite la obtención de perfiles electroforéticos unidimensionales característicos de algunas especies de triatominos. Sin embargo, el reporte de los patrones electroforéticos de proteínas salivales de las especies de Rhodnius ha sido escaso.

Objetivo. Hacer un análisis comparativo de los perfiles electroforéticos unidimensionales de las proteínas salivales de $R$. colombiensis, $R$. pallescens, $R$. pictipes, $R$. prolixus y $R$. robustus. Materiales y métodos. Se obtuvieron los perfiles electroforéticos de la saliva de las especies en estudio mediante electroforesis en gel de poliacrilamida con dodecilsulfato sódico (Sodium Dodecyl Sulfate Polyacrylamide Gel Electrophoresis, SDS-PAGE) y se construyó un fenograma mediante el método UPGMA (Unweighted Pair Group Method Using Arithmetic Averages).

Resultados. Los perfiles electroforéticos de las proteínas solubles de saliva presentaron bandas en un rango de masa aproximado de 15 a $45 \mathrm{kDa}$, los cuales permitieron diferenciar las cinco especies estudiadas. El fenograma reveló la existencia de dos grupos principales: uno conformado por los grupos cisandinos Pictipes y Prolixus y otro constituido por el grupo transandino Pallescens.

Conclusiones. Existen diferencias en los perfiles electroforéticos de las proteínas salivales entre $R$. colombiensis, $R$. pallescens, $R$. pictipes, $R$. prolixus y R. robustus, cuya variabilidad permitió construir un fenograma congruente con los grupos del género Rhodnius.

Recibido: 08/04/2019

Aceptado: $27 / 11 / 2019$

Publicado: 04/12/2019

Citación:

Meneses A, Rodríguez CC, Suárez Y, Carranza JC, Vallejo GA. Los patrones electroforéticos de proteínas salivales permiten diferenciar los grupos transandino y cisandino de las especies de Rhodnius de Colombia. Biomédica. 2020;40:404-11. https://doi.org/10.7705/biomedica.4992

\section{Correspondencia:}

Gustavo Adolfo Vallejo, Laboratorio de Investigaciones en Parasitología Tropical, Facultad de Ciencias, Universidad del Tolima, apartado aéreo 546 , Ibagué, Colombia

Teléfono: (578) 277 1212, extensión 9348

gvallejo @ut.edu.co

\section{Contribución de los autores:}

Arlid Meneses: obtención de muestras, trabajo de laboratorio, análisis de datos y revisión de resultados Cristian Camilo Rodríguez: trabajo de laboratorio Yazmín Suárez: orientación del trabajo de laboratorio y análisis de datos

Julio César Carranza: orientación del trabajo de laboratorio y revisión de resultados

Gustavo Adolfo Vallejo: análisis de datos y revisión de resultados

Todos los autores participaron en la elaboración del manuscrito.

\section{Financiación:}

Este trabajo fue financiado por el Fondo de Investigaciones de la Universidad del Tolima, proyecto No. 320120516.

Conflicto de intereses:

Los autores declaran no tener conflicto de intereses.
Palabras clave: Rhodnius; proteínas y péptidos salivales; electroforesis en gel de poliacrilamida.

\section{Salivary proteins electrophoretic patterns enabled differentiating Colombian Rhodnius Trans-Andean and Cis-Andean groups.}

Introduction: Rhodnius (Hemiptera: Reduviidae: Triatominae) species are made up of haematophagous insect vectors for Trypanosoma cruzi (Chagas' disease aetiological agent) and T. rangeli, an infective parasite that is not pathogenic for vertebrate hosts. The study of their salivary protein diversity enables the obtention of characteristic one-dimensional electrophoretic profiles of some triatomine species; however, few reports have dealt with Rhodnius species salivary proteins electrophoretic patterns.

Objective: To compare R. colombiensis, R. pallescens, R. pictipes, R. prolixus, and $R$. robustus' salivary proteins one-dimensional electrophoretic profiles.

Materials and methods: SDS-PAGE was used for obtaining electrophoretic profiles of saliva from the species under study. The unweighted pair group method with arithmetic mean (UPGMA) was used for constructing a phenogram.

Results: Electrophoretic profiles of soluble saliva had protein bands ranging from 15 to 45 $\mathrm{kDa}$, thereby enabling the five species studied to be differentiated. The phenogram revealed two main groups, one formed by the Pictipes and Prolixus cis-Andean groups and another consisting of the Pallescens trans-Andean group.

Conclusion: Differences were revealed regarding $R$. colombiensis, $R$. pallescens, $R$. pictipes, $R$. prolixus, and $R$. robustus electrophoretic profiles of salivary proteins; their variability facilitated constructing a phenogram which was taxonomically congruent with the groups from the genus Rhodnius.

Keywords: Rhodnius; salivary proteins and peptides; electrophoresis, polyacrylamide gel.

El género Rhodnius está compuesto por 21 especies de insectos hematófagos, muchas de ellas indistinguibles morfológicamente entre sí $(1,2)$. Todas las especies de este género son vectores de Trypanosoma 
cruzi, agente causal de la enfermedad de Chagas, y de T. rangeli, el cual es infectivo, pero no patógeno para los vertebrados $(3,4)$. Aunque $T$. rangeli podría encontrarse en el intestino de cualquier triatomino, solo en las especies de Rhodnius logran infectar las glándulas salivales. Se les considera como los vectores biológicos de este parásito y se ha observado que algunos de sus genotipos son patógenos para algunas especies de Rhodnius (4).

Debido a sus características morfológicas, de comportamiento, biogeográficas y genéticas, el género Rhodnius parece ser un taxón monofilético compuesto por tres grupos: el grupo Prolixus, integrado por $R$. barreti, $R$. dalessandroi, $R$. domesticus, $R$. milesi, $R$. marabaensis, $R$. montenegrensis, $R$. nasutus, $R$. neglectus, $R$. prolixus y $R$. taquarusuensis; el grupo Pictipes, conformado por $R$. amazonicus, $R$. brethesi, $R$. paraensis, $R$. piptipes, $R$. stali y $R$. zeledoni, y el grupo Pallescens, del cual hacen parte $R$. colombiensis, $R$. ecuadoriensis y R. pallescens. Los grupos Prolixus y Piptipes se distribuyen al oriente de la cordillera de los Andes y se les denomina cisandinos, en tanto que el grupo Pallescens se encuentra al occidente de la cordillera de los Andes y recibe el nombre de transandino (5-9).

En estudios previos se ha reportado que con el perfil electroforético de las hemoproteínas (nitroforinas) se pueden diferenciar las especies fenotípicamente similares (10-12). Asimismo, se ha evidenciado que los patrones electroforéticos de las proteínas salivales solubles bajo condiciones desnaturalizantes (SDSPAGE) permiten diferenciar entre especies de triatominos (13-15). Sin embargo, la información disponible sobre los perfiles electroforéticos unidimensionales de la saliva de las especies de Rhodnius es limitada.

Por esta razón, en el presente estudio se hizo un análisis comparativo de los perfiles electroforéticos bajo condiciones desnaturalizantes de proteínas salivales solubles de $R$. colombiensis, $R$. pallescens, $R$. pictipes, $R$. prolixus y R. robustus.

\section{Materiales y métodos}

Las glándulas salivales se obtuvieron a partir de la disección de ninfas de quinto estadio $\left(\mathrm{N}_{5}\right)$ de colonias de R.colombiensis, R. prolixus, R. pallescens, $R$. pictipes y $R$. robustus establecidas en el insectario del Laboratorio de Investigaciones en Parasitología Tropical de la Universidad del Tolima (cuadro 1). Se emplearon como mínimo 10 ejemplares de cada especie en cada réplica biológica y ensayo electroforético.

Cuadro 1. Colonias de las especies de Rhodnius empleadas según su procedencia

\begin{tabular}{lll}
\hline Especie & \multicolumn{1}{c}{ Procedencia } & $\begin{array}{l}\text { Ambiente } \\
\text { de captura }\end{array}$ \\
\hline R. colombiensis & Chaparral, Tolima & Silvestre \\
& Coyaima, Tolima & Silvestre \\
& lbagué, Tolima & Silvestre \\
& Icononzo, Tolima & Silvestre \\
R.pano, Tolima & Silvestre \\
R.pictipes & San Sebastián de Buenavista, Magdalena & Silvestre \\
R.prolixus & Amazonas, municipio desconocido & Silvestre \\
& Boyacá, municipio desconocido & Doméstico \\
& Coyaima, Tolima & Doméstico \\
& Medina, Cundinamarca & Doméstico \\
& Santander, municipio desconocido & Doméstico \\
& Sierra Nevada de Santa Marta, Magdalena & Doméstico \\
R. robustus & Villanueva, Casanare & Silvestre \\
\hline
\end{tabular}


Después de la disección, las glándulas se lavaron tres veces en solución fisiológica fría ( $\mathrm{NaCl} 0,9 \%$ ), se recolectaron en un microtubo en cama de hielo y se suspendieron de nuevo en solución fisiológica fría en una proporción de $2 \mu \mathrm{l}$ por cada par de glándulas. Para permitir la extravasación de la saliva, las glándulas se perforaron con un alfiler entomológico, se centrifugaron a 9.000 r.p.m. durante 5 minutos a $4{ }^{\circ} \mathrm{C}$ y el sobrenadante se transfirió a un nuevo microtubo. La concentración de proteínas solubles totales se determinó por espectrofotometría mediante el método de Bradford (16).

Se empleó $1 \mu \mathrm{g}$ de proteína salival soluble para cada electroforesis y estas se corrieron en geles de resolución de poliacrilamida al $12 \%$ acoplados a un gel de $5 \%$ de concentración usando el sistema Mini PROTEAN Tetra Cell ${ }^{\mathrm{TM}}$ (Bio-Rad). El corrido electroforético se hizo a $90 \mathrm{~V}$ empleando tampón de Trisglicina (Tris, $25 \mathrm{mM}$, glicina, $192 \mathrm{mM}$, SDS 0,1 \% p/v) durante 2 horas. Los geles se colorearon con nitrato de plata $(17,18)$. Con el objetivo de comprobar la reproducibilidad del polimorfismo observado en la saliva de $R$. prolixus, se hicieron seis réplicas biológicas con sus respectivos geles electroforéticos, en tanto que para las demás especies se hicieron tres.

Los geles de SDS-PAGE se digitalizaron empleando un fotodocumentador Gel Doc XR+System ${ }^{\text {TM }}$ (Bio-Rad). Los pesos moleculares de las bandas de proteínas visualizadas se calcularon con el programa Image Lab, versión 5.2.1, y una matriz de caracteres y taxones se elaboró con base en la presencia o ausencia de bandas para calcular el coeficiente de similitud de Nei-Li-Dice (19) y construir un fenograma empleando el método UPGMA.

\section{Resultados}

Los perfiles electroforéticos de la saliva de $R$.colombiensis, $R$. pallescens, $R$. pictipes, $R$. prolixus y $R$. robustus mostraron una compleja composición proteica de bajo peso molecular (figura 1), cuyos perfiles fueron similares a los descritos previamente en otras especies de triatominos (13-15), en tanto que las bandas de proteínas tuvieron pesos moleculares menores a $45 \mathrm{KDa}$, la mayoría de ellas en un rango de 15 a $25 \mathrm{KDa}$. Rhodnius pictipes presentó el mayor número de bandas exclusivas y 22 de ellas eran compartidas, por lo menos, por dos especies. Las poblaciones de R. prolixus de Boyacá y Tolima presentaron el mayor número de bandas de proteínas (14), y las de R. colombiensis de Coyaima y Chaparral presentaron el menor número de bandas (cuadro 2).
A.

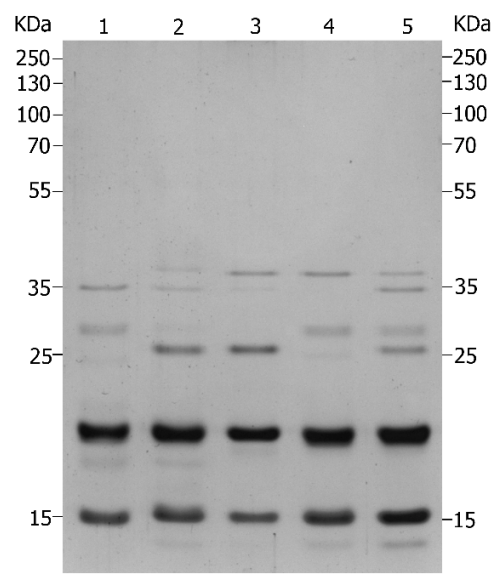

B.

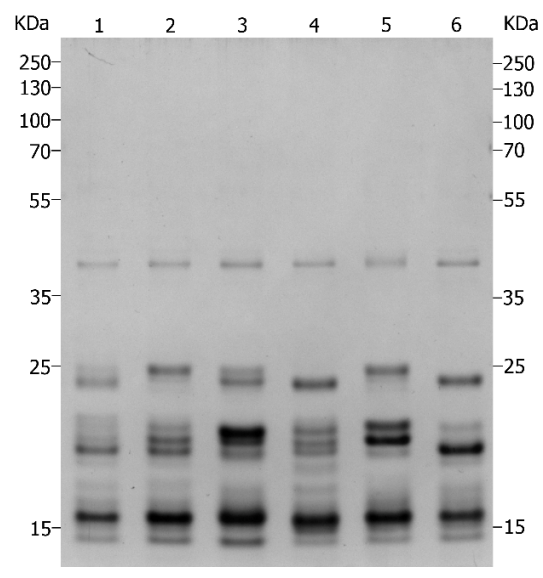

C.

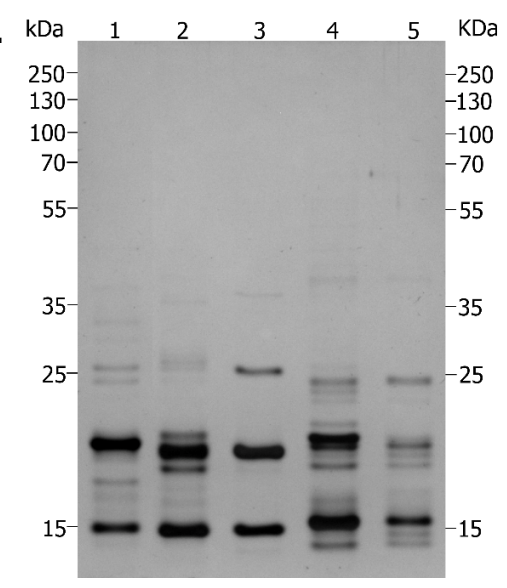

Figura 1. Proteínas salivales en geles de SDS-PAGE al $12 \%$, coloreados con nitrato de plata. A) Especies del género Rhodnius: (1) $R$. picitipes, (2) R. pallescens, (3) R. colombiensis (Coyaima, Tolima), (4) R. prolixus (Boyacá), (5) R. robustus (Putumayo). B) Colonias de R. prolixus: (1) Cundinamarca, (2) Tolima, (3) Boyacá, (4) Casanare, (5) Magdalena y (6) Santander. C) Colonias de R. colombiensis: (1) lbagué, (2) Icononzo, (3) Coyaima, (4) Chaparral y (5) Líbano 
Con el fenograma construido mediante el método UPGMA se diferenciaron cada una de las especies estudiadas y se evidenció la existencia de dos grupos principales (figura 2): uno conformado por $R$. robustus, $R$. prolixus y $R$. pictipes y, el otro, por R. pallescens y R. colombiensis.

Cuadro 2. Pesos moleculares en KDa de las bandas de proteínas salivales de cinco especies del género Rhodnius

\begin{tabular}{|c|c|c|c|c|c|c|c|c|c|c|c|c|c|c|c|}
\hline \multicolumn{2}{|c|}{ Banda } & \multirow{2}{*}{$\begin{array}{c}R . \\
\text { pictipes }\end{array}$} & \multirow{2}{*}{$\begin{array}{c}R . \\
\text { pallescens }\end{array}$} & \multicolumn{5}{|c|}{ R. colombiensis } & \multirow{2}{*}{$\begin{array}{c}R . \\
\text { robustus }\end{array}$} & \multicolumn{6}{|c|}{ R. prolixus } \\
\hline $\mathbf{N}^{\circ}$ & MW & & & Ibagué & Incononzo & Coyaima & Chaparral & Líbano & & Boyacá & Magdalena & Santander & Cundinamarca & Tolima & Casanare \\
\hline$\overline{1}$ & $42,31 \pm 0,37$ & 0 & 0 & 0 & 0 & 0 & 0 & 0 & 0 & 1 & 1 & 1 & 1 & 1 & 1 \\
\hline 2 & $40,07 \pm 0,31$ & 0 & 0 & 0 & 0 & 0 & 0 & 0 & 1 & 1 & 1 & 1 & 1 & 1 & 1 \\
\hline 3 & $38,03 \pm 0,0$ & 1 & 0 & 0 & 0 & 0 & 0 & 0 & 0 & 0 & 0 & 0 & 0 & 0 & 0 \\
\hline 4 & $37,33 \pm 0,38$ & 0 & 0 & 0 & 1 & 1 & 1 & 1 & 0 & 0 & 0 & 0 & 0 & 0 & 0 \\
\hline 5 & $35,61 \pm 0,0$ & 0 & 1 & 0 & 0 & 0 & 0 & 0 & 0 & 0 & 0 & 0 & 0 & 0 & 0 \\
\hline 6 & $34,49 \pm 0,07$ & 0 & 0 & 1 & 1 & 1 & 0 & 1 & 0 & 0 & 0 & 0 & 0 & 0 & 0 \\
\hline 7 & $32,26 \pm 0,0$ & 1 & 0 & 0 & 0 & 0 & 0 & 0 & 0 & 0 & 0 & 0 & 0 & 0 & 0 \\
\hline 8 & $29,63 \pm 0,0$ & 1 & 0 & 0 & 0 & 0 & 0 & 0 & 0 & 0 & 0 & 0 & 0 & 0 & 0 \\
\hline 9 & $28,19 \pm 0,13$ & 0 & 0 & 1 & 1 & 0 & 1 & 1 & 0 & 0 & 0 & 0 & 0 & 0 & 0 \\
\hline 10 & $27,26 \pm 0,0$ & 0 & 0 & 0 & 0 & 0 & 0 & 0 & 0 & 0 & 1 & 0 & 0 & 0 & 0 \\
\hline 11 & $26,52 \pm 0,0$ & 0 & 1 & 0 & 0 & 0 & 0 & 0 & 0 & 0 & 0 & 0 & 0 & 0 & 0 \\
\hline 12 & $25,89 \pm 0,0$ & 1 & 0 & 0 & 0 & 0 & 0 & 0 & 0 & 0 & 0 & 0 & 0 & 0 & 0 \\
\hline 13 & $25,59 \pm 0,01$ & 0 & 0 & 0 & 1 & 1 & 0 & 1 & 0 & 0 & 0 & 0 & 0 & 0 & 0 \\
\hline 14 & $24,62 \pm 0,15$ & 1 & 0 & 1 & 0 & 0 & 1 & 0 & 1 & 1 & 1 & 0 & 1 & 1 & 0 \\
\hline 15 & $23,8 \pm 0,20$ & 0 & 0 & 0 & 0 & 0 & 0 & 0 & 0 & 1 & 1 & 1 & 1 & 1 & 1 \\
\hline 16 & $23,05 \pm 0,18$ & 0 & 0 & 0 & 0 & 0 & 0 & 0 & 1 & 1 & 0 & 0 & 0 & 0 & 0 \\
\hline 17 & $21,3 \pm 0,11$ & 0 & 0 & 1 & 0 & 0 & 0 & 0 & 0 & 1 & 0 & 0 & 1 & 1 & 1 \\
\hline 18 & $20,35 \pm 0,05$ & 0 & 1 & 0 & 0 & 0 & 0 & 0 & 0 & 1 & 1 & 0 & 0 & 0 & 0 \\
\hline 19 & $19,93 \pm 0,13$ & 1 & 0 & 0 & 0 & 0 & 0 & 0 & 1 & 1 & 1 & 1 & 1 & 0 & 1 \\
\hline 20 & $19,5 \pm 0,07$ & 0 & 1 & 1 & 1 & 1 & 1 & 1 & 0 & 0 & 0 & 0 & 0 & 1 & 1 \\
\hline 21 & $19,1 \pm 0,04$ & 0 & 0 & 0 & 0 & 0 & 0 & 0 & 1 & 1 & 0 & 1 & 1 & 1 & 1 \\
\hline 22 & $18,48 \pm 0,08$ & 0 & 0 & 0 & 0 & 0 & 0 & 0 & 1 & 1 & 1 & 0 & 0 & 1 & 0 \\
\hline 23 & $18,06 \pm 0,22$ & 0 & 1 & 0 & 0 & 0 & 0 & 0 & 0 & 0 & 0 & 1 & 1 & 1 & 1 \\
\hline 24 & $17,68 \pm 0,13$ & 1 & 0 & 1 & 1 & 0 & 0 & 0 & 0 & 0 & 0 & 0 & 0 & 0 & 0 \\
\hline 25 & $17,17 \pm 0,0$ & 0 & 1 & 0 & 0 & 0 & 0 & 0 & 0 & 0 & 0 & 0 & 0 & 0 & 0 \\
\hline 26 & $16,76 \pm 0,19$ & 0 & 0 & 0 & 0 & 0 & 0 & 0 & 1 & 1 & 1 & 0 & 1 & 1 & 1 \\
\hline 27 & $16,23 \pm 0,28$ & 1 & 1 & 0 & 0 & 0 & 0 & 0 & 0 & 1 & 1 & 1 & 1 & 1 & 0 \\
\hline 28 & $15,43 \pm 0,06$ & 0 & 0 & 0 & 0 & 0 & 0 & 0 & 1 & 1 & 1 & 1 & 1 & 1 & 1 \\
\hline 29 & $15,04 \pm 0,47$ & 1 & 1 & 1 & 1 & 1 & 1 & 1 & 1 & 0 & 1 & 1 & 1 & 1 & 1 \\
\hline 30 & $<15$ & 1 & 0 & 0 & 1 & 1 & 1 & 1 & 1 & 1 & 1 & 1 & 1 & 1 & 1 \\
\hline
\end{tabular}

MW: Molecular weight (peso molecular) en kDa

Significado de los números: 0 , banda ausente, 1 , banda presente

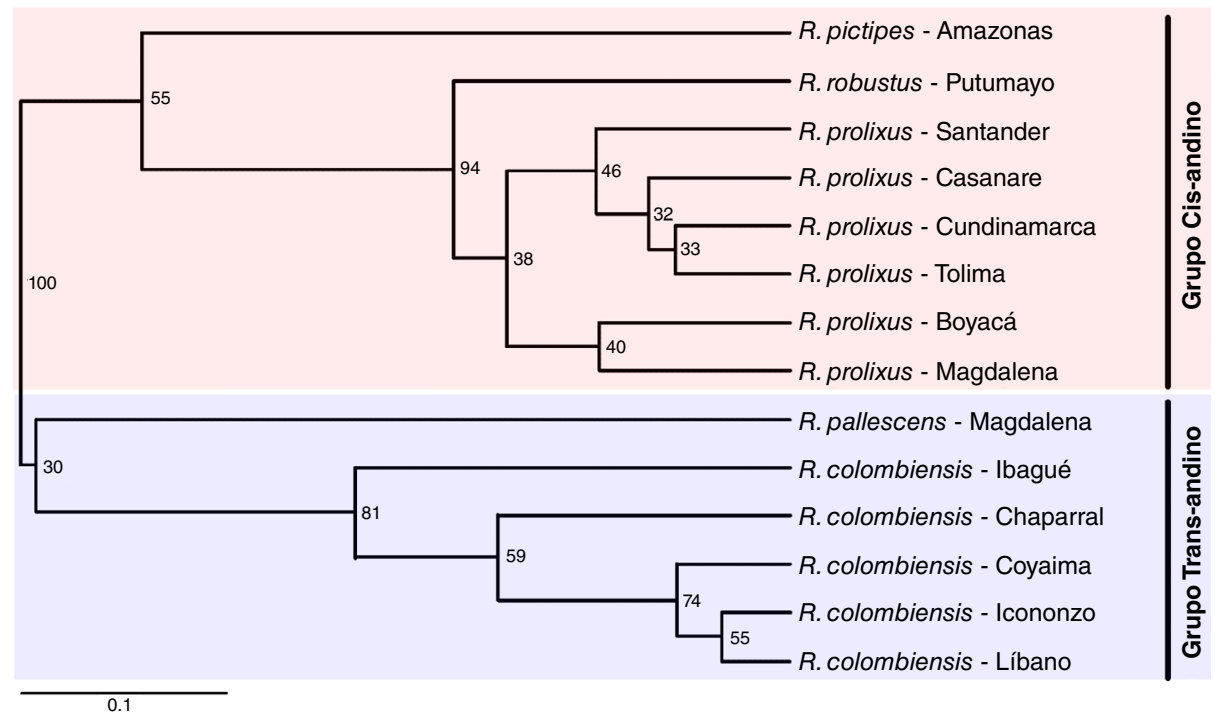

Figura 2. Fenograma construido por el método UPGMA a partir de perfiles electroforéticos de proteínas salivales de cinco especies del género Rhodnius. La escala horizontal representa el índice de similitud derivado del índice de Dice. 


\section{Discusión}

Los pesos moleculares de las bandas de las proteínas salivales de $R$. colombiensis, $R$. pallescens, $R$. pictipes, $R$. prolixus y $R$. robustus obtenidos mediante SDS-PAGE concordaron con las masas moleculares de las proteínas codificadas por secuencias de ADNc de longitud completa de las glándulas salivales de $R$. prolixus (20), con algunas proteínas de la familia de las lipocalinas caracterizadas como nitroforinas, con proteínas de unión a amina biogénica y con inhibidores de agregación plaquetaria (21-23), así como con los pesos moleculares de la mayoría de las proteínas identificadas en la saliva de $R$. neglectus, $R$. prolixus, $R$. robustus y $R$. brethesi (24-26). La función principal de estas proteínas es contrarrestar los eventos hemostáticos del huésped, tales como la vasoconstricción, la agregación plaquetaria y la coagulación sanguínea, así como la inflamación y las reacciones del sistema inmunitario del huésped, permitiéndole al insecto un flujo constante de sangre durante su alimentación $(27,28)$.

Estos resultados concuerdan con lo reportado por diferentes autores, quienes mencionan que los perfiles electroforéticos de saliva obtenidos mediante SDS-PAGE de proteínas solubles y electroforesis de hemoproteínas salivales permiten identificar los triatominos hasta el nivel de especie (10$12,14,15)$. La técnica aplicada también puede servir para ayudar a caracterizar las especies del género Rhodnius que son morfológicamente similares.

La variabilidad electroforética de las proteínas salivales en la SDSPAGE en poblaciones de una misma especie, pero proveniente de áreas geográficas diferentes se ha estudiado en Panstrongylus megistus de Brasil (13) y Triatoma dimidiata de Colombia y Guatemala $(14,15)$. Los resultados de estos estudios concuerdan con lo aquí evidenciado en el sentido de que no fue posible establecer una relación entre el polimorfismo observado y el área geográfica o el grado de asociación con las viviendas humanas. No obstante, se han reportado diferencias en los perfiles electroforéticos de hemoproteínas salivales en $R$. prolixus provenientes de Venezuela y la región central de Colombia, lo que evidencia que la variación de la composición proteica puede reflejar distancias geográficas entre las poblaciones de una misma especie $(10,11)$. Es probable que esta relación entre el polimorfismo y la región geográfica pueda observarse al aumentar la escala geográfica y comparar los perfiles electroforéticos de diferentes poblaciones de países de Centroamérica y Suramérica.

Por otra parte, la posición de R. pictipes del grupo Pictipes en la misma rama con $R$. prolixus y $R$. robustus, del grupo Prolixus, muestra la cercanía entre los dos grupos; sin embargo, la filogenia de R. pictipes y las demás especies del grupo ha sido tema de controversia, ya que debido a su amplia distribución geográfica y a las características ecológicas y las similitudes morfológicas con otros Triatominae y predadores redúvidos no compartidas por la mayoría de las especies de la tribu Rhodniini, $R$. pictipes podría ser la especie más cercana a la forma ancestral del género Rhodnius $(8,29,30)$. En estudios de filogenia molecular basados en marcadores nucleares y mitocondriales, los resultados sobre la posición filogenética de $R$. pictipes han diferido. Se ha encontrado que el grupo transandino Pallescens está estrechamente relacionado con el grupo Pictipes $(30,31)$, en tanto que otros autores reportan que $R$. pictipes está más cerca a $R$. prolixus que a las especies del grupo transandino $(1,32,33)$. 
Desde otra perspectiva, en los estudios sobre la interacción de Rhodnius y $T$. rangeli se ha postulado una asociación entre los grupos del primero y los genotipos del segundo, observándose que el grupo transandino Pallescens transmite KP1(-) únicamente por inoculación a T. rangeli, mientras que el grupo Prolixus solo transmite KP1(+) a T. rangeli (4). Recientemente, se ha reportado que las glándulas salivales de $R$. pictipes pueden ser infectadas por la cepa SC-58 de T. rangeli KP1(-). Por lo tanto, R. pictipes podría transmitir el genotipo KP1(-) de T. rangeli, exhibiendo así características biológicas similares a las evidenciadas en las especies del grupo transandino (34).

Puede concluirse que los perfiles electroforéticos de las proteínas salivales permitieron diferenciar entre $R$. colombiensis, $R$. pallescens, $R$. pictipes, $R$. prolixus y $R$. robustus, y que la variabilidad electroforética interespecífica proporcionó datos para la generación de un fenograma congruente con los grupos transandino y cisandino. Asimismo, la información obtenida en este estudio indica que $R$. pictipes puede compartir características fenotípicas con los grupos Pallescens y Prolixus, lo que respalda el estatus de $R$. pictipes como la especie más cercana a la forma ancestral del género Rhodnius.

\section{Referencias}

1. Justi S, Galvão C, Schrago G. Geological changes of the Americas and their influence on the diversification of the neotropical kissing bugs (Hemiptera: Reduviidae: Triatominae). PLoS Negl Trop Dis. 2016;10:e0004527. https://doi.org/10.1371/journal.pntd.0004527

2. Da Rosa J, Justino H, Nascimento J, Mendonça V, Rocha C, de Carvalho D, et al. A new species of Rhodnius from Brazil (Hemiptera, Reduviidae, Triatominae). ZooKeys. 2017;675:125. https://doi.org/10.3897/zookeys.675.12024

3. Cuba-Cuba C. Revisión de los aspectos biológicos y diagnósticos del Trypanosoma (Herpetosoma) rangeli. Rev Soc Bras Med Trop. 1998;31:207-20. https://doi.org/10.1590/S0037-86821998000200007

4. Vallejo G, Suárez Y, Olaya J, Gutiérrez S, Carranza J. Trypanosoma rangeli: un protozoo infectivo y no patógeno para el humano que contribuye al entendimiento de la transmisión vectorial y la infección por Trypanosoma cruzi, agente causal de la enfermedad de Chagas. Revista de la Academia Colombiana de Ciencias Exactas Físicas y Naturales. 2015;39:111-22.

5. Abad-Franch F, Monteiro F, Gurgel-Golçalves R, Dias F, Diotaiuti L. Ecology, evolution, and the long-term surveillance of vector-borne Chagas disease: A multi-scale appraisal of the tribe Rhodniini (Triatominae). Acta Trop. 2009;110:159-77. https://doi.org/10.1016/j.actatropica.2008.06.005

6. Díaz S, Panzera F, Jaramillo-O N, Pérez R, Fernández R, Vallejo G, et al. Genetic, cytogenetic and morphological trends in the evolution of the Rhodnius (Triatominae: Rhodniini) TransAndean group. PLoS One. 2014;9:e87493. https://doi.org/10.1371/journal.pone.0087493

7. Galvão C, Justi S. The evolutionary origin of diversity in Chagas disease vectors. Trends Parasitol. 2017;33:42-52. https://doi.org/10.1016/j.pt.2016.11.002

8. Schofield C, Dujardin J. Theories on the evolution of Rhodnius. Actualidades Biológicas. 1999;21:183-97.

9. Schofield C, Galvão C. Classification, evolution and species groups within the Triatominae. Acta Trop. 2009;110:88-100. https://doi.org/10.1016/j.actatropica.2009.01.010

10. Arévalo A, Carranza JC, Guhl F, Vallejo GA. Patrones electroforéticos de hemoproteínas salivares (nitroforinas) de Rhodnius colombiensis y Rhodnius prolixus (Hemiptera, Reduviidae, Triatominae). Biomédica. 2007;27(Suppl.1):137-42. https://doi.org/10.7705/biomedica.v27i1.257

11. Soares R, Gontijo N, Romanha A, Diotaiuti L, Pereira M. Salivary heme proteins distinguish Rhodnius prolixus from Rhodnius robustus (Hemiptera: Reduviidae: Triatominae). Acta Trop. 1998;71:285-91. https://doi.org/10.1016/S0001-706X(98)00065-5

12. Soares R, Sant'Anna M, Gontijo N, Romanha A, Diotaiuti L, Pereira M. Identification of morphologically similar Rhodnius species (Hemiptera: Reduviidae: Triatominae) by electrophoresis of salivary heme proteins. Am J Trop Med Hyg. 2000;62:157-61. 
13. Barbosa S, Diotaiuti L, Braga E, Pereira M. Variability of the salivary proteins of 20 Brazilian populations of Panstrongylus megistus (Hemiptera: Reduviidae: Triatominae). Acta Trop. 2004;92:25-33. https://doi.org/10.1016/j.actatropica.2004.05.012

14. Flórez M, Niño R, Gonzalo R, Muñoz G, Angulo V. Perfil electroforético de proteínas presentes en la saliva de Triatoma dimidiata (Hemiptera: Reduviidae:Triatominae). Salud UIS. 2009;41:121-7.

15. Pineda S, Melgar S, Dorn P, Agreda E, Rodas A, Monroy C. Salivary protein profiles distinguish triatomine species and populations of Triatoma dimidiata (Hemiptera: Reduviidae). J Med Entomol. 2008;45:52-8. https://doi.org/10.1093/jmedent/45.1.52

16. Bradford MM. A rapid and sensitive method for the quantitation of microgram quantities of protein utilizing the principle of protein-dye binding. Anal Biochem. 1976;72:248-54. https://doi.org/10.1016/0003-2697(76)90527-3

17. Blum $\mathrm{H}$, Beier $\mathrm{H}$, Gross HJ. Improved silver staining of plant proteins, RNA and DNA in polyacrylamide gels. Electrophoresis. 1987;8:93-9.

18. Shevchenko A, Wilm M, Vorm O, Mann M. Mass spectrometric sequencing of proteins silverstained polyacrylamide gels. Anal Chem. 1996;68:850-8. https://doi.org/10.1021/ac950914h

19. Dice L. Measures of the amount of ecological association between species. Ecology. 1945;26:297-302. https://doi.org/10.2307/1932409

20. Ribeiro J, Andersen J, Silva-Neto M, Pham V, Garfield M, Valenzuela J. Exploring the sialome of the blood-sucking bug Rhodnius prolixus. Insect Biochem Mol Biol. 2004;34:61-79. https://doi.org/10.1016/j.ibmb.2003.09.004

21. Andersen J, Gudderra N, Francischetti I, Ribeiro J. The role of salivary lipocalins in blood feeding by Rhodnius prolixus. Arch Insect Biochem Physiol. 2005;58:97-105. https://doi.org/10.1002/arch.20032

22. Francischetti I, Ribeiro J, Champagne D, Andersen J. Purification, cloning, expression, and mechanism of action of a novel platelet aggregation inhibitor from the salivary gland of the blood-sucking bug, Rhodnius prolixus. J Biol Chem. 2000;275:12639-50. https://doi.org/10.1074/jbc.275.17.12639

23. Sun J, Yuda M, Miura K, Chinzei Y. Characterization and cDNA cloning of a hemoprotein in the salivary glands of the blood-sucking insect, Rhodnius prolixus. Insect Biochem Mol Biol. 1998;29:191-200. https://doi.org/10.1016/S0965-1748(97)00115-X

24. Costa C, Sousa M, Ricart C, Santana J, Teixeira A, Roepstorff P, et al. 2-DE-based proteomic investigation of the saliva of the Amazonian triatomine vectors of Chagas disease: Rhodnius brethesi and Rhodnius robustus. J Proteomics. 2011;74:1652-63. https://doi.org/10.1016/j.jprot.2011.02.022

25. Montandon C, Barros E, Vidigal P, Mendes M, Anhê A, de Oliveira H, et al. Comparative proteomic analysis of the saliva of the Rhodnius prolixus, Triatoma lecticularia and Panstrongylus herreri triatomines reveals a high interespecific functional biodiversity. Insect Biochem Mol Biol. 2016;71:83-90. https://doi.org/10.1016/j.ibmb.2016.02.009

26. Santiago P, Assumpção T, Araújo C, Bastos I, Neves D, Silva I, et al. A deep insight into the sialome of Rhodnius neglectus, a vector of Chagas disease. PLoS Negl Trop Dis. 2016;10:e0004581. https://doi.org/10.1371/journal.pntd.0004581

27. Andrade B, Teixeira R, Barral A, Barral-Netto M. Haematophagous arthropod saliva and host defense system: A tale of tear and blood. Anais da Academia Brasileira de Ciências. 2005;77:665-93. https://doi.org/10.1590/S0001-37652005000400008

28. Fontaine A, Diouf I, Bakkali N, Missé D, Pagès F, Fusai F, et al. Implication of haematophagous arthropod salivary proteins in host-vector interactions. Parasit Vectors. 2011;187:1-17. https://doi.org/10.1186/1756-3305-4-187.

29. Bargues M, Schofield C, Dujardin J. Classification and phylogeny of the Triatominae. En: Telleria J, Tibayrenc M, editors. American Trypanosomiasis, Chagas Disease One Hundred Years of Research. Montpellier: Elsevier; 2010. p.117-47. https://doi.org/10.1016/B978-0-12-384876-5.00006-X

30. Lyman D, Monteiro F, Escalante A, Cordon-Rosales C, Wesson D, Dujardin JP, et al. Mitochondrial DNA sequence variation among triatomine vectors of Chagas' disease. Am J Trop Med Hyg. 1999;60:377-86. https://doi.org/10.4269/ajtmh.1999.60.377

31. Monteiro F, Wesson D, Dotson E, Schofield C, Beard C. Phylogeny and molecular taxonomy of the Rhodniini derived from mitochondrial and nuclear DNA sequences. Am J Trop Med Hyg. 2000;62:460-5. 
32. Hypsa V, Tietz D, Zrzavy J, Rego R, Galvão C, Jurberg J. Phylogeny and biogeography of Triatominae (Hemiptera Reduviidae): Molecular evidence of a New World origin of the Asiatic clade. Mol Phylogen Evol. 2002;23:447-57. https://doi.org/10.1016/S1055-7903(02)00023-4

33. Justi S, Russo C, Dos Santos J, Takashi M, Galvão C. Molecular phylogeny of Triatomini (Hemiptera: Reduviidae: Triatominae). Parasit Vectors. 2014;7:149-61. https://doi.org/10.1186/1756-3305-7-149

34. Barreto-Santana D, Santos-Schuenker L, da Fonseca A, Gurgel-Gonçalves R, Cuba-Cuba A. Susceptibility of different Rhodnius species (Hemiptera, Reduviidae, Triatominae) to a Brazilian strain of Trypanosoma rangeli (SC58/KP1-). Biomédica. 2015;35:81-9. https://doi.org/10.7705/biomedica.v35i1.2213 